\title{
Antibiotic Resistance, Virulence Factors and Phylogenetic Analysis of Efflux Proteins of Coagulase Negative Staphylococcus Isolates from Sewage Samples
}

\author{
Kannan Balachander, Deborah Gnana Selvam Alexander* \\ Post-Graduate Department of Microbiology, The American College, Madurai, India
}

Email address:

deborahalexander12@gmail.com (D. G. S. Alexander)

${ }^{*}$ Corresponding author

\section{To cite this article:}

Kannan Balachander, Deborah Gnana Selvam Alexander. Antibiotic Resistance, Virulence Factors and Phylogenetic Analysis of Efflux Proteins of Coagulase Negative Staphylococcus Isolates from Sewage Samples. Pharmaceutical Science and Technology.

Vol. 5, No. 1, 2021, pp. 14-23. doi: 10.11648/j.pst.20210501.13

Received: February 24, 2021; Accepted: March 26, 2021; Published: May 26, 2021

\begin{abstract}
Multidrug-resistant pathogens are now emergent worldwide and pose a serious threat to disease prevention and treatment. The World Health Organization declared anti-microbial resistance as a major peril to human beings. Staphylococcus is part of the normal microbiome of humans and are responsible for opportunistic infections. Like S. aureus, Coagulasenegative staphylococcus are also clinically important as the causal agents of severe diseases, nosocomial infections, catheterassociated infection, bacteremia, septicemia. Methicillin resistant Staphylococci species are widely known and methicillin resistant CoNS, known as MR-CoNS, have been found to carry the mecA gene. Various virulence factors help these organisms in their pathogenicity and antibiotic-resistance is aided by the presence of efflux pumps. In our study, we have isolated 45 isolates from sewage water which were tentatively identified as CoNS based on biochemical characteristics. Then virulence and antibiotics susceptibility patterns were screened by standard protocols. Multiple drug resistant CoNS were found in our study and in silico analysis of an annotated protein sequence of the efflux pump SepA of Pseudomonas putida was carried out. Sequence analysis of the efflux protein gene revealed that it is phylogenetically related to the AcrA of Staphylococcus sp. and a RND transporter of Vibrio sp. The widespread presence of MR-CoNS is a cause for serious concern as sewage treatment plants are reservoirs for the spread of antibiotic resistance.
\end{abstract}

Keywords: Coagulase Negative Staphylococcus, Efflux Proteins, Multiple Drug Resistant Organism, Phylogenetic Analysis

\section{Introduction}

The staphylococci are gram-positive microorganisms, the size around $0.5 \mu \mathrm{m}-1 \mu \mathrm{m}$ in diameter. They are observed as clusters under a microscope. Occasionally it can be seen in short chains. They are ubiquitous, mostly found in human skin and mucus membranes. Staphylococci are salt-tolerant and they can grow in $7.5 \% \mathrm{NaCl}$. Staphylococci are often hemolytic. Coagulase test helps to distinguish the $S$. aureus from other staphylococci organisms and some of $S$. aureus from environmental isolates are incapable of producing coagulase. In the genus Staphylococcus, the $S$. aureus and $S$. intermedius are the only coagulase-positive organisms and all other species are coagulase-negative. Staphylococcus aureus is a common microflora found on our skin surfaces and nasal passages of healthy human beings. Approximately $25-40 \%$ of the human population is colonized with $S$. aureus organism [1]. MR-CoNS harboring the mecA gene, which helps resist methicillin and other $\beta$ - lactam antimicrobials, are referred to as methicillin-resistant Staphylococcus (MR-CoNS). Methicillin-resistant Staphylococcus (MR-CoNS) is a dangerous pathogen that causes infection in various parts of the human body. It causes mild infections on the skin, like sores, boil, or abscesses, but it can also cause severe infections such as infection of the bloodstream, surgical wounds, the lungs, or urinary tract infections. Most MR-CoNS infections are not serious, some can be life-threatening [2]. Since MRCoNS infections are difficult to treat. Methicillin-resistant Staphylococcus aureus also causes severe infection, which is different from MR-CoNS. Staphylococcus resistance to 
methicillin was reported soon after its introduction in October 1960 [3]. MR- CoNS is now endemic in India. The incidence of methicillin-resistant Staphylococcus varies from 50\% in South India [4] to $25 \%$ in the western part of India [5]. CAMRSA has been increasingly were reported in India [6]. In India, bloodstream infections (BSI) are caused by Staphylococcus species and CoNS are also involved in causing BSI. Those infections are acquired in healthcare settings. $S$. epidermidis are most frequently involved in BSI in developing countries [7].

Antibiotics are widely used to treat numerous infectious diseases. As a result, antibiotic resistant bacteria are also rampant. According to the Centre for Disease Control, at least 2.8 million people in the United States become infected with antibiotic-resistant bacteria each year, with the mortality reaching 35,000 as a direct result of such infections [8]. The role of wastewater as an environmental reservoir in the creation and spread of antibiotic resistance is vital [9]. Particularly when a huge percentage of antibiotics end up in the environment [10]. This includes $\beta$-lactam antibiotics, which have been isolated from both soil and drainage systems [11]. In both hospital and wastewater drainage systems, mecA gene has been detected [12]. Both culture-based techniques and molecular analysis has found MRSA has been found in municipal water [13]. In the present study, we isolated and identified the CoNS from a municipal Waste Water Treatment Plant WWTP, Madurai, and screened the isolates for multiple antibiotic resistance and compared it with other studies from the locality and other Indian strains. These isolates were tested for antibiotic susceptibility. With the help of sequences obtained from BacMet, an in silico sequence analysis of the SepA superfamily of efflux protein was carried out.

\section{Material and Methods}

\subsection{Sample Collection and Processing}

The wastewater treatment plant at Avaniyapuram, Madurai was first started in 1924. It receives wastewater from the south zone of the river Vaigai. It is one of the largest plants in Madurai. At present, the plant receives 15 to 20Mld (million liters per day) of wastewater from the main pumping station at Santhaipettai, and the daily discharge depends upon the availability of electricity and capacity of the machinery. One hundred milliliters of wastewater were collected in autoclaved glass conical flask at Avaniyapuram wastewater plant and the samples were transported within $1 \mathrm{~h}$ to the laboratory in an icebox. We collected the wastewater sample once in September 2019, December 2019, and February 2020 .

\subsection{Sample Processing and Isolation}

Collected wastewater samples were centrifuged in sterile centrifuge tubes at $8000 \mathrm{rpm}$ for 30 minutes at $4^{\circ} \mathrm{C}$. Supernatant was discarded and the pellet was collected. The pellet was inoculated into the Nutrient broth with $7.5 \% \mathrm{NaCl}$ for enrichment process [14] and incubated at $37^{\circ} \mathrm{C}$ for overnight incubation. A loopful of culture from the nutrient broth was streaked onto sterile Mannitol Salt Agar and Blood agar plates aseptically and incubated at $37^{\circ} \mathrm{C}$ for $18-24 \mathrm{~h}$.

\subsection{Biochemical Characterization and Detection of Virulence Factors of Staphylococcus sp.}

The cultured isolates were performed various biochemical tests such as gram staining, coagulase, catalase, oxidase, and TSI test. The virulence factors were screened by various tests such as DNase, hemolysis, starch hydrolysis, and biofilm identification by congo red test.

\subsection{Antibiotic Susceptibility Test}

Antibiotic susceptibility was performed by the Kirby Bauer method which is a standard diffusion procedure and helps to determine the susceptibility of isolated bacteria to different antibiotics. The filter paper discs are impregnated with antibiotics of specific concentrations. Mueller-Hinton agar plates were used in this procedure and overnight broth cultures of the bacterial isolates were swabbed into the surface of the medium and antibiotic discs were placed on the seeded surface. After incubation, the zone of inhibition was formed. The measuring of the zone of inhibition helps to interpret the results based on the standard chart developed by The Clinical \& Laboratory Standards Institute. Twenty-five of the isolates were subjected to antibiotic susceptibility screening.

\subsection{Phylogenetic Analysis}

SepA is an efflux pump which belongs to the Resistance Nodulation cell Division (RND) superfamily of efflux proteins. It is present in the chromosome of Pseudomonas sp. involved in the efflux of multiple drugs. It contains 382 amino acids. This sequence was retrieved from BacMet (antibacterial biocide and metal resistance gene database) and was aligned with other sequences by protein BLAST.

\section{Results}

The sewage wastewater sample was collected at the Avaniyapuram sewage water treatment plant in a sterile autoclaved conical flask. Before sample collection, $\mathrm{pH}$ and temperature were measured in situ.

Table 1. pH and temperature of the sample collection from the sewage plant.

\begin{tabular}{lll}
\hline Sample collection period & $\mathbf{p H}$ & Temperature \\
\hline September -2019 & 8.5 & $37^{\circ} \mathrm{C}$ \\
December -2019 & 9 & $38.5^{\circ} \mathrm{C}$ \\
February -2020 & 8.5 & $35.8^{\circ} \mathrm{C}$ \\
\hline
\end{tabular}

After overnight incubation, nutrient broth showing turbidity was taken as positive for presumptive staphylococci. Then a loopful of nutrient broth culture was taken and streaked (quadrant streaking) onto the MSA plate and blood agar plate aseptically. Both plates were incubated for $24 \mathrm{~h}$ at room temperature. After incubation, it was observed that there were too many colonies on the MSA 
plate, which turned from red to yellow color showing that the isolates utilize mannitol as a source of carbon. In the blood agar plate, the colonies have grown appeared white and some of the colonies were $\beta$ - hemolytic. Then 45 single colonies were chosen for maintenance as pure cultures and further characterization. The selected isolates were streaked using sterile inoculation needle onto Nutrient Agar slants supplemented with $7.5 \% \mathrm{NaCl}$ and after growth was observed, stored at RT.

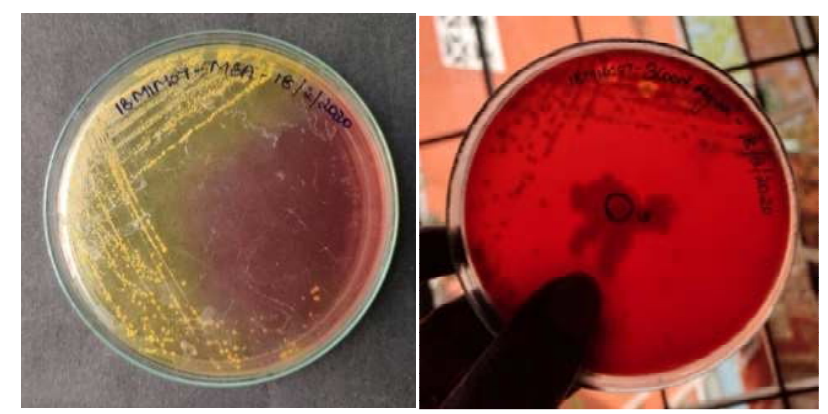

Figure 1. MSA and Blood agar plate showing the growth of presumptive Staphylococci.

Most of the isolates are purple indicating gram-positive organisms. They were found to be cocci and were seen in clusters. Out of the 45 isolates, 41 were found to be grampositive cocci. $41 / 45 \times 100=91.11 \%$. Therefore, $91 \%$ of the isolates were gram-positive, cocci, and cluster-forming bacteria and tentatively identified as Staphylococcus sp.

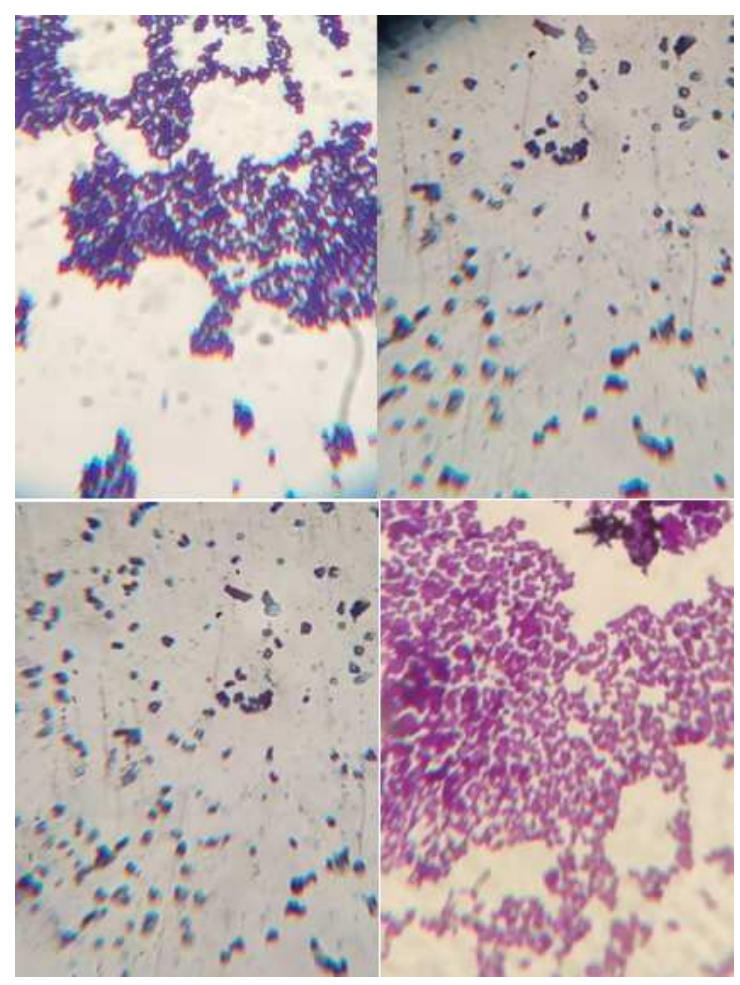

Figure 2. Gram staining images under light microscope 100x.

\subsection{Biochemical Tests}

The preliminary biochemical tests performed were oxidase and catalase tests. Oxidase test was carried out by disc method which is quick with the results was observed within few seconds. 36 of the isolates are oxidase negative, whereas the other 9 isolates are oxidase-positive which produced purple color after they were smeared on the disc. In catalase test, all of the isolates are produces bubbles, so all isolates are presence of catalase. It is a distinguishing feature that separates Staphylococci from Streptococci which are catalase negative.

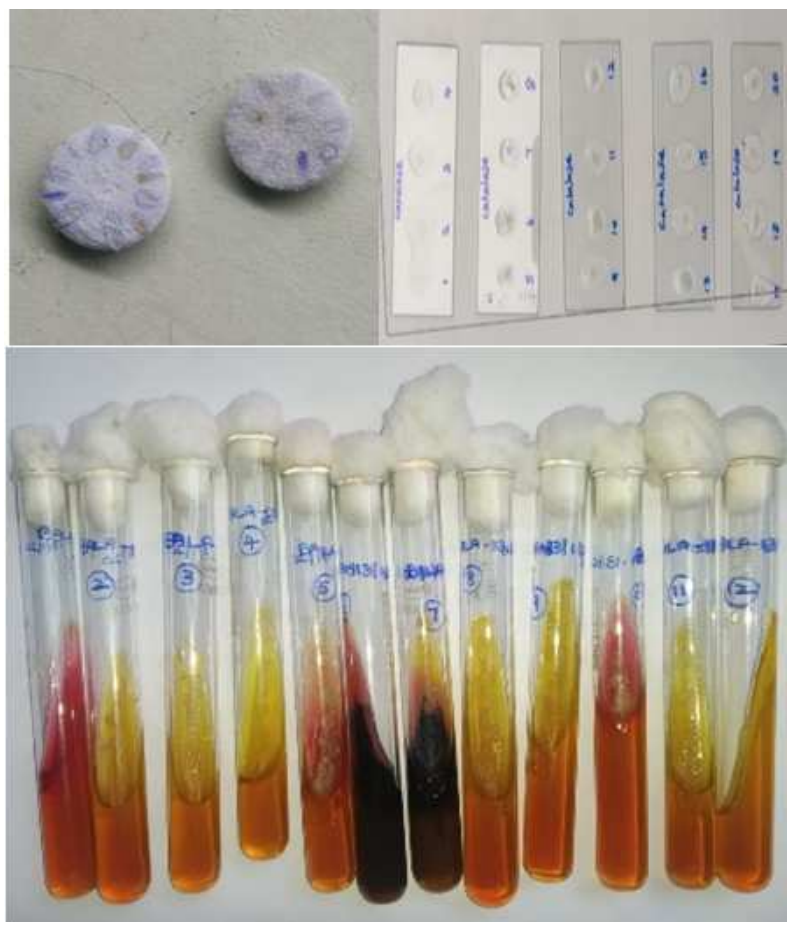

Figure 3. Oxidase, Catalase and TSI test result.

In the tube coagulase test, after inoculation, the tube was observed respectively in $2 \mathrm{~h}, 4 \mathrm{~h}, 6 \mathrm{~h}, 8 \mathrm{~h}$, and up to $24 \mathrm{~h}$. However, no clotting was observed; all isolates are coagulase-negative. After incubation of TSI agar, color changes were observed in the butt, slant and gas production were observed; of the 25 isolates, 22 isolates utilized 3 sugars and others are not.

\subsection{Virulence Factor Test}

In the Starch hydrolysis test, following incubation, iodine solution was poured on the surface of the starch medium; zone of clearance representing starch hydrolysis was observed around 2 isolates which represents positive result and all other 43 isolates are negative for amylase. For the detection of DNase production, following incubation, $1 \mathrm{~N}$ $\mathrm{HCl}$ solution was flooded on the surface of DNase medium and observe for the presence of a clear zone around the isolates; all isolates were negative for the presence of DNase. For hemolysis test, after $24 \mathrm{~h}$ incubation, the plates were checked for the presence of clear zones; all isolates were negative for hemolysis. Similarly, none of the isolates was capable of biofilm formation which was checked by Congo red plate method. 


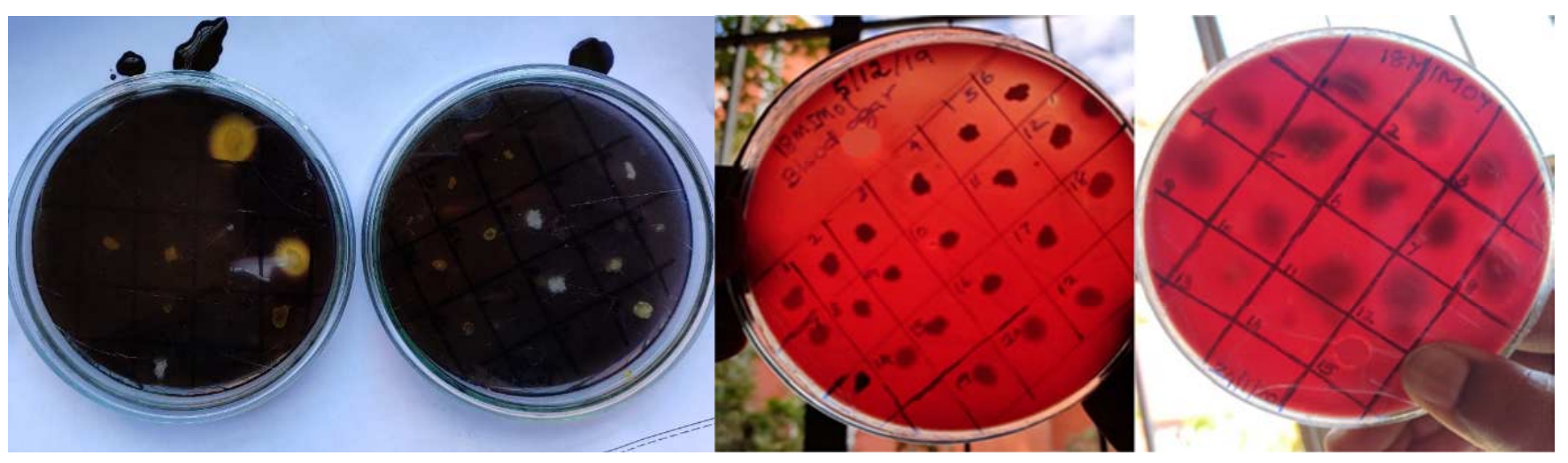

Figure 4. Starch hydrolysis and Hemolysis activity.

Table 2. Biochemical test results of isolated CoNS organisms.

\begin{tabular}{|c|c|c|c|c|c|c|c|c|c|}
\hline Colony no & Gram staining & Oxidase & Catalase & Coagulase & DNase & TSI & Starch hydrolysis & Biofilm formation & Hemolysis \\
\hline 1. & Positive & Negative & Positive & Negative & Negative & - & Negative & Negative & $\gamma$ - Hemolysis \\
\hline 2. & Positive & Negative & Positive & Negative & Negative & - & Negative & Negative & $\gamma$ - Hemolysis \\
\hline 3. & Negative & Negative & Positive & Negative & Negative & - & Negative & Negative & $\gamma$ - Hemolysis \\
\hline 4. & Positive & Negative & Positive & Negative & Negative & - & Negative & Negative & $\gamma$ - Hemolysis \\
\hline 5. & Positive & Negative & Positive & Negative & Negative & - & Negative & Negative & $\gamma$ - Hemolysis \\
\hline 6. & Positive & Negative & Positive & Negative & Negative & - & Negative & Negative & $\gamma$ - Hemolysis \\
\hline 7. & Negative & Negative & Positive & Negative & Negative & - & Negative & Negative & $\gamma$ - Hemolysis \\
\hline 8. & Negative & Negative & Positive & Negative & Negative & - & Negative & Negative & $\gamma$ - Hemolysis \\
\hline 9. & Positive & Negative & Positive & Negative & Negative & - & Negative & Negative & $\gamma$ - Hemolysis \\
\hline 10. & Positive & Positive & Positive & Negative & Negative & - & Negative & Negative & $\gamma$ - Hemolysis \\
\hline 11. & Positive & Negative & Positive & Negative & Negative & - & Negative & Negative & $\gamma$ - Hemolysis \\
\hline 12. & Positive & Negative & Positive & Negative & Negative & - & Negative & Negative & $\gamma$ - Hemolysis \\
\hline 13. & Positive & Negative & Positive & Negative & Negative & - & Negative & Negative & $\gamma$ - Hemolysis \\
\hline 14. & Positive & Negative & Positive & Negative & Negative & - & Negative & Negative & $\gamma$ - Hemolysis \\
\hline 15. & Positive & Negative & Positive & Negative & Negative & - & Negative & Negative & $\gamma$ - Hemolysis \\
\hline 17. & Positive & Negative & Positive & Negative & Negative & - & Negative & Negative & $\gamma$ - Hemolysis \\
\hline 18. & Positive & Negative & Positive & Negative & Negative & - & Negative & Negative & $\gamma$-Hemolysis \\
\hline 19. & Positive & Negative & Positive & Negative & Negative & - & Negative & Negative & $\gamma$ - Hemolysis \\
\hline 20. & Negative & Negative & Positive & Negative & Negative & - & Negative & Negative & $\gamma$ - Hemolysis \\
\hline 21. & Positive & Negative & Positive & Negative & Negative & Positive & Negative & Negative & $\gamma$ - Hemolysis \\
\hline 22. & Positive & Negative & Positive & Negative & Negative & Positive & Negative & Negative & $\gamma$ - Hemolysis \\
\hline 23. & Positive & Positive & Positive & Negative & Negative & Positive & Negative & Negative & $\gamma$ - Hemolysis \\
\hline 24. & Positive & Positive & Positive & Negative & Negative & Negative & Positive & Negative & $\gamma$ - Hemolysis \\
\hline 25. & Positive & Positive & Positive & Negative & Negative & Negative & Negative & Negative & $\gamma$ - Hemolysis \\
\hline 26. & Positive & Negative & Positive & Negative & Negative & Positive & Negative & Negative & $\gamma$ - Hemolysis \\
\hline 27. & Positive & Positive & Positive & Negative & Negative & Positive & Negative & Negative & $\gamma$ - Hemolysis \\
\hline 28. & Positive & Positive & Positive & Negative & Negative & Negative & Negative & Negative & $\gamma$ - Hemolysis \\
\hline 29. & Positive & Positive & Positive & Negative & Negative & Positive & Negative & Negative & $\gamma$ - Hemolysis \\
\hline 30. & Positive & Positive & Positive & Negative & Negative & Positive & Positive & Negative & $\gamma$ - Hemolysis \\
\hline 33. & Positive & Negative & Positive & Negative & Negative & Positive & Negative & Negative & $\gamma$ - Hemolysis \\
\hline 34. & Positive & Negative & Positive & Negative & Negative & Positive & Negative & Negative & $\gamma$ - Hemolysis \\
\hline 35. & Positive & Negative & Positive & Negative & Negative & Positive & Negative & Negative & $\gamma$ - Hemolysis \\
\hline 36. & Positive & Negative & Positive & Negative & Negative & Positive & Negative & Negative & $\gamma$-Hemolysis \\
\hline 37. & Positive & Negative & Positive & Negative & Negative & Positive & Negative & Negative & $\gamma$ - Hemolysis \\
\hline 38. & Positive & Negative & Positive & Negative & Negative & Positive & Negative & Negative & $\gamma$ - Hemolysis \\
\hline 39. & Positive & Negative & Positive & Negative & Negative & Positive & Negative & Negative & $\gamma$ - Hemolysis \\
\hline 40. & Positive & Negative & Positive & Negative & Negative & Positive & Negative & Negative & $\gamma$ - Hemolysis \\
\hline 41. & Positive & Negative & Positive & Negative & Negative & Positive & Negative & Negative & $\gamma$ - Hemolysis \\
\hline 42. & Positive & Negative & Positive & Negative & Negative & Positive & Negative & Negative & $\gamma$ - Hemolysis \\
\hline 43. & Positive & Negative & Positive & Negative & Negative & Positive & Negative & Negative & $\gamma$ - Hemolysis \\
\hline 44. & Positive & Negative & Positive & Negative & Negative & Positive & Negative & Negative & $\gamma$ - Hemolysis \\
\hline 45. & Positive & Negative & Positive & Negative & Negative & Positive & Negative & Negative & $\gamma$-Hemolysis \\
\hline
\end{tabular}




\subsection{Antibiotic Susceptibility Test}

Twenty isolates were subjected to antibiotic susceptibility test (Kirby Bauer method). After incubation, the zone inhibition was measured and identified whether they were sensitive or resistant based on CLSI standards. All 20 isolates are resistant to methicillin; 6 isolates are resistant to

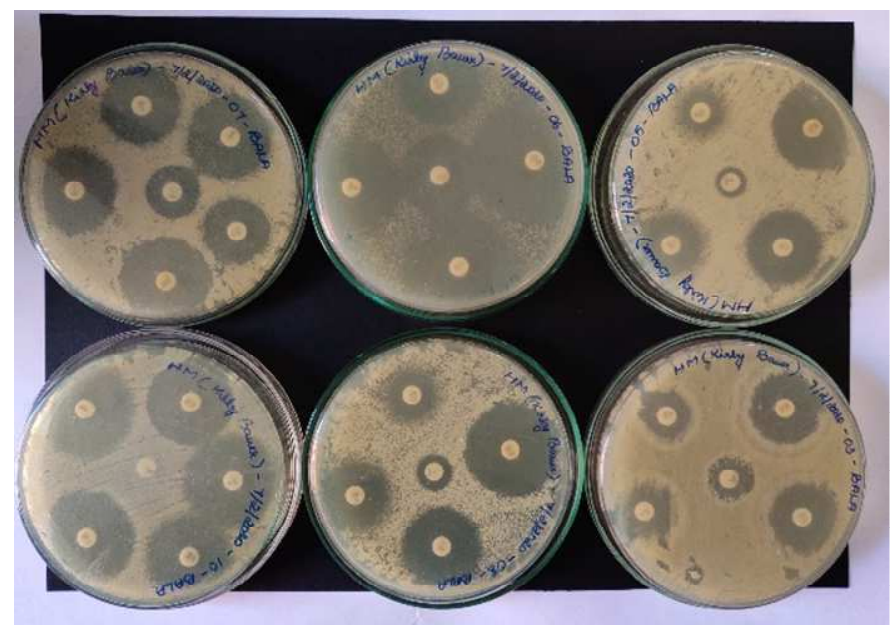

norfloxacin and the other 14 were sensitive. One culture was resistant to chloramphenicol and the other 19 are sensitive. Thirteen isolates were sensitive to ciprofloxacin, 6 are intermediate and one was resistant. All isolates were sensitive to tetracycline and levofloxacin.

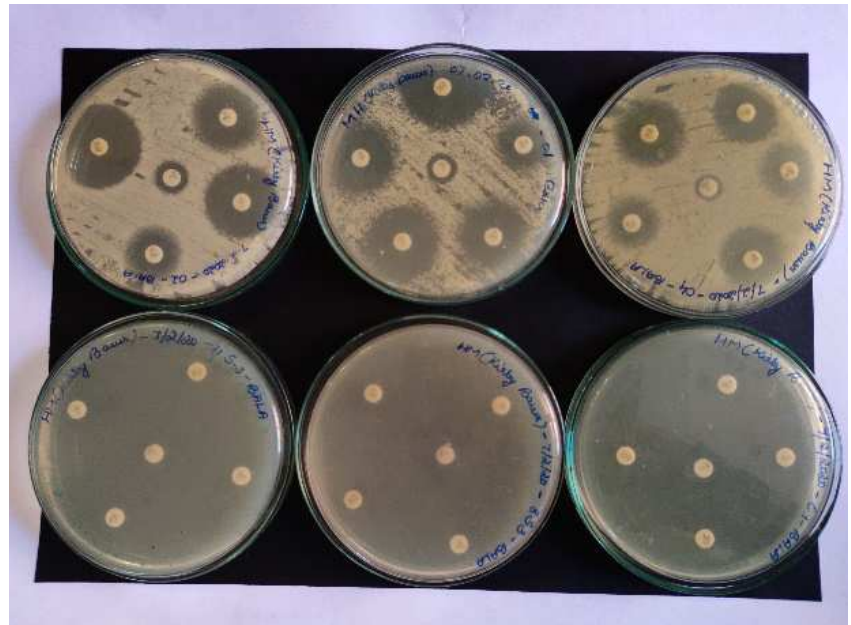

Figure 5. Antibiotic Susceptibility Test (Kirby Bauer method).

Table 3. Antibiotic Resistance Profile of CoNS isolated from sewage water samples.

\begin{tabular}{|c|c|c|c|c|c|c|}
\hline Colony no & $\begin{array}{l}\text { Methicillin } \\
(5 \mu \mathrm{g})\end{array}$ & $\begin{array}{l}\text { Tetracycline } \\
(30 \mu \mathrm{g})\end{array}$ & $\begin{array}{l}\text { Norfloxacin } \\
(10 \mu \mathrm{g})\end{array}$ & $\begin{array}{l}\text { Levofloxacin } \\
(5 \mu \mathrm{g})\end{array}$ & $\begin{array}{l}\text { Chloramphenicol } \\
(30 \mu \mathrm{g})\end{array}$ & $\begin{array}{l}\text { Ciprofloxacin } \\
(5 \mu \mathrm{g})\end{array}$ \\
\hline 1. & $\mathrm{R}$ & $S$ & $S$ & $S$ & $S$ & $S$ \\
\hline 2. & $\mathrm{R}$ & $S$ & $S$ & $S$ & $S$ & $\mathrm{~S}$ \\
\hline 3. & $\mathrm{R}$ & S & S & S & S & S \\
\hline 4. & $\mathrm{R}$ & $\mathrm{S}$ & $\mathrm{S}$ & $\mathrm{S}$ & S & $\mathrm{S}$ \\
\hline 5. & $\mathrm{R}$ & $S$ & $S$ & $S$ & $S$ & $\mathrm{~S}$ \\
\hline 6. & $\mathrm{R}$ & S & $\mathrm{R}$ & S & $\mathrm{R}$ & I \\
\hline 7. & $\mathrm{R}$ & S & $\mathrm{S}$ & $\mathrm{S}$ & S & S \\
\hline 8. & $\mathrm{R}$ & $S$ & $S$ & $S$ & $S$ & I \\
\hline 9. & $\mathrm{R}$ & S & S & S & S & I \\
\hline 10. & $\mathrm{R}$ & S & S & S & S & $\mathrm{S}$ \\
\hline 11. & $\mathrm{R}$ & S & S & S & S & $\mathrm{S}$ \\
\hline 12. & $\mathrm{R}$ & S & $\mathrm{R}$ & S & S & I \\
\hline 13. & $\mathrm{R}$ & S & $\mathrm{R}$ & $\mathrm{S}$ & S & $\mathrm{R}$ \\
\hline 14. & $\mathrm{R}$ & S & $\mathrm{R}$ & S & S & I \\
\hline 15. & $\mathrm{R}$ & S & $\mathrm{R}$ & S & S & I \\
\hline 16. & $\mathrm{R}$ & $\mathrm{S}$ & $\mathrm{S}$ & $\mathrm{S}$ & $\mathrm{S}$ & $\mathrm{S}$ \\
\hline 17. & $\mathrm{R}$ & S & S & S & S & S \\
\hline 18. & $\mathrm{R}$ & S & $\mathrm{R}$ & S & S & S \\
\hline 19. & $\mathrm{R}$ & $\mathrm{S}$ & $\mathrm{R}$ & $\mathrm{S}$ & S & S \\
\hline 20. & $\mathrm{R}$ & S & S & S & S & S \\
\hline
\end{tabular}

R- Resistant, S- Sensitive, I- Intermediate.

\subsection{Phylogenetic Analysis}

Sequence analysis of the SepA (of Pseudomonas putida) efflux protein with other sequences revealed that it is homologous to various efflux proteins like AcrA of Staphylococcus sp. The tree shows that this SepA protein is also homologous to another resistance efflux protein called RND transporter of Vibrio $s p$. The phylogenetic analysis showed that these proteins have evolved from a common ancestral gene. 
WP 188351763-1-16-151-efflux-RND-transporter-periplasmic-adaptor-subunit-partial-

Staphylococcus-aureus-

RAV24677-1-12-92-multidrugtransporter-subunit-MdtA-partial-

Staphylococcus-warneri-

WP 164648279-1-5-371-efflux-RND-transporter-periplasmic-adaptor-subunit-

Vibrio-astriarenae-

WP 136487242-1-5-374-efflux-RND transporter-periplasmic-adaptor-subunit-

Vibrio-sp-H11-

WP 143680947-1-5-372-efflux-RND-transporter-periplasmic-adaptor-subunit-

Vibrio-fumissii-

WP 075989105-1-5-374-efflux-RND-transporter-periplasmic-adaptor-subunit-

Vibriofluvialis-

WP 032081444-1-5-374-efflux-RND-transporter-periplasmic-adaptor-subunitVibriofluvialis-

WP 104966308-1-5-374-efflux-RND transporter-periplasmic-adaptor-subunit-

Vibrio-fluvialis-

WP 061057031-1-5-374-efflux-RND transporter-periplasmic-adaptor-subunit-

Vibrio-fluvialis-

WP 020433573-1-5-374-efflux RND-transporter-periplasmic-adaptor-subunit-

Vibrio-fluvialis-

WP 158151584-1-5-374-efflux RND-transporter-periplasmic-adaptor-subunit-

Vibrio-fluvialis-

sp-Q849Q9-SEPA PSEP1-Probable-efflux-pump-periplasmic-linker-protein-SepA-OS Pseudomonasputida-strain-F1-/-ATCC-700007-GN sepA-PE 3-SV 1 WP 142302492-1-1-60-biotinflipoyl-binding-protein-partial-

Staphylococcus-hominis-

KKF71116-1-3-374-multidrug-transporter-Vibrio-

parahaemolyticus-

RXS10582-1-3-120-multidrug-efflux RND-transporter-periplasmic-adaptor-subunit-AcrA-partial-

St aphylococcus-saprophyticus-

RAV24666-1-3-88-efflux-transporter-periplasmic-adaptor-subunit-partial-

Staphylococcus-wameri-

RIM18497-1-3-125-efflux-RND transporter-periplasmic-adaptor-subunit-partial-

Staphylococcus-chromogenes-

IEES94089-1-1-71-acriflavine-resistance-protein-A-domain-prot ein-partial-Staphylococcus-aureus-subspaureus-USA300 TCH959-

WP 142389283-1-1-148-efflux-RND-transporter-periplasmic-adaptor-subunit-partial-

Staphylococcus-aureus-

NGB74349-1-8-344 efflux-RND-transporter-periplasmic-adaptor-subunit -

Staphylococcus-aureus-

KKF68901-1-15-379-multidrug-transporter-Vibrio-

lparahaemolyticus-

[MRF64731-1-1-80-multidrug-efflux-RND-transporter-periplasmic-adaptor-subunit-AcrE-

StaphylococcusepidermidisKEKS1000-1-1-177 efflux-transporter-RND-family-MFP-subunit-partial-Staphylococcusaureus-1801-1-2010WP 006712328-1-13-371-efflux-RND-transporter-periplasmic-adaptor-subunit-

Vibrio-ichthyoenteri-

WP 046874751-1-11-375-efflux-RND-transporter-periplasmic-adaptor-subunit-

Vibrio-diabolicus-

WP 152812457-1-11-375-efflux-RND transporter-periplasmic-adaptor-subunit-

Vibrio-sp-VGm-2-

WP 145474267-1-11-375-efflux-RND-transporter-periplasmic-adaptor-subunit-

Vibrio-sp-ES-044-

WP 109168511-1-11-375-efflux-RND-transporter-periplasmic-adaptor-

subunit-Vibrio-sp-T9-

WP 005390787-1-11-375-MULTISECIES-efflux-RND-transporter-periplasmic-adaptor-

subunit-Vibrio-

WP 182032606-1-11-375 efflux-RND-transporter-periplasmic-adaptor-subunit-

Vibrio-diabolicus-

WP 164666211-1-11-375 efflux-RND-transporter-periplasmic-adaptor-subunit-

Vibrio-diabolicus-

WP 053807186-1-11-375-MULTISPECIES-efflux-RND-transporter-periplasmic-adaptor-subunit-Vibrio-

diabolicus-subgroup-

W P 006742894-1-11-375-efflux RND-transporter-periplasmic-adaptor-subunit-

Vibrio-antiquarius-

WP 047008568-1-11-371-efflux-RND-transporter-periplasmic-adaptor-subunit-

Vibrio-diabolicus-

WP 065645378-1-11-371-efflux-RND-transporter-periplasmic-adaptor-subunit-

Vibrio-diabolicus-

WP 182037023-1-11-375-efflux-RND transporter-periplasmic-adaptor-subunit-

Vibrio-diabolicus-

WP 042763416-1-11-375-efflux-RND -transporter-periplasmic-adaptor-subunit-Vibrio-

parahaemolyticus-

Figure 6. Phylogenetic tree showing the relationship between SepA probable efflux pump periplasmic linker protein of Pseudomonas putida with the multidrug - efflux RND transporter periplasmic adaptor subunit AcrA of Staphylococcus sp. 


\section{Discussion}

The CoNS are mostly associated with clinical device infections as they readily form biofilms on the surface of invasive devices like a catheter. The CoNS are important in nosocomial infections and other clinical infections. CoNS are ubiquitous; they are mostly found on skin surfaces and mucus membranes. Most of them are opportunistic pathogens. These organisms can be found as normal microflora on every human being. Staphylococci are often hemolytic and cause disease in humans and animals. Mostly they cause wound infections, bloodstream infections, and hospital-acquired infections such as surgical wound infections. The CoNS S. epidermidis causes the infection associated with indwelling medical devices. The $S$. saprophyticus causes UTI infections in girls. The other Staphylococcal organisms such as $S$. warneri, $S$. haemolyticus, S. intermedius, and S. schleiferi are also pathogenic which cause diseases infrequently in humans $[15$, $16]$.

Sewage water includes a wide variety of microorganisms such as bacteria, viruses, and protozoa. Most microorganisms in wastewater are pathogenic and highly virulent. Wastewater can contain a lot of opportunistic pathogens (e.g., Escherichia coli, Enterobacter cloacae, Enterococcus faecalis, Klebsiella pneumoniae, Proteus vulgaris, or Pseudomonas aeruginosa) which can cause various systemic infections, particularly among people with poor and impaired immune systems. Salmonella and Shigella, or enteropathogenic strains of Escherichia coli, responsible for gastroenteritis, salmonellosis, shigellosis, can also be present in wastewater $[16,17]$.

CoNS $S$. pasteuri was identified in a significant proportion (65.7\%) of drinking water supply $S$. carnosus, $S$. condimenti, S. equorum, S. piscifermentans, S. succinus, and S. xylosus representing staphylococcal organisms linked to fermented foods [18]. S. epidermidis and other CoNS are generally known as non-pathogenic having little virulence factors. The most frequently appearing infectious agent is the incremental clarification of microbial pathogenesis related to the involvement of foreign species in the organism and especially concerning S. epidermidis [19]. Staphylococci are the leading cause of hospital and community-acquired infections, particularly antibiotic-resistant strains. These bacteria are ubiquitous and commonly spread in a wide range of environments, with exceptional survival. In addition to serving as a source for the diffusion of resistance genes to various bacteria, including pathogens, they can survive for long periods in diverse environments. Their capacity for survival and gain resiliency genes can partially enlighten why they remain to be a noteworthy human and animal pathogen [2].

CoNS can be characterized by various tests, such as colony morphology, catalase test, gram staining, slide and tube coagulase test (i.e., bound coagulase and free coagulase) (read after 4, $24 \mathrm{~h}$ ) and mannitol salt agar, deoxyribonuclease test. The biochemical tests include novobiocin sensitivity, mannitol utilization, ornithine decarboxylation, urease production, phosphatase test, polymyxin B disc test, VogesProskauer test, mannose fermentation, trehalose fermentation [20]. In our study most of the above-mentioned tests were performed for the tentative identification of CoNS.

Bacteria produce extracellular enzymes or exoenzymes, which help them in the invasion of the host. These enzymes act as virulence factors making the strains pathogenic. During an infection, bacterial survival is a mechanism dependent on the organism's ability to evade and bypass host defense strategies. S. aureus synthesizes a large number of toxins and exoproteins. Nearly all synthesizes a lot of enzymes and cytotoxins, including four hemolysins (alpha, beta, gamma, and delta), collagenase, lipases, nucleases, hyaluronidase, and proteases [21]. These proteins can principally have the role of transforming host tissues into growth nutrients necessary for the survival of the ivading bacteria. Some strains also synthesize other extra exoproteins such as the toxic shock syndrome toxin-1 (TSST 1) (SEA, SEB, SEC, SED, SEE, SEG, SEH, and SEI), and the toxins A and B. Gammahemolysine is involved in the lysis of mammalian erythrocytes. These toxins also affect neutrophils and macrophages. Coagulase produced by Staphylococcus sp. Interferes with blood clotting by binding prothrombin to a complex called staphylothrombin in the host. These are some of the strategies adapted by $S$. aureus cells defend themselves from the host's immune response [22].

Hydrolytic amylases ( $\alpha$-amylase, $\beta$-amylase, and glucoamylase) of starch are among the most widely used in modern biotechnology. Fungi and bacteria secrete amylases to convert excess extracellular starch into sugars. Staphylococcus aureus is well known as a major human Gram-positive pathogen that produces multiple surface and secretory proteins, including separate enzymes and pathogenic factors, supporting colonization and host-tissue infection. $\alpha$-amylase is an enzyme separated from $S$. aureus that catalysts the colonization and survival [23].

Hemolysin is one of the virulence factors observed in bth coagulase-positive and coagulase-negative staphylococci (CoNS). There were substantial differences in non-hemolytic isolates between CoNS, CoPS, and all non-hemolytic isolates are part of CoNS. Most of the Staphylococcus isolated from cattle exhibited delta hemolysin on sheep's blood agar plate, while most humans developed alpha-hemolysin. $10(50 \%)$ of the 20 human isolates developed double hemolysin (DH), while the DH-ratio for bovine isolates (one out of 20) was slightly low) [24].

In our studies, we screened all CoNS isolates for hemolytic activity in the blood agar supplemented with human blood. All isolates weren't able to produce hemolysis; so, all are non-hemolytic CoNS.

In recent decades, CoNS has been continuously decreasing resistance to most of the antibiotics accessible. In specific penicillin, oxacillin, ciprofloxacin, clindamycin, erythromycin, or gentamicin have established significant and 
radical changes in the quantity of resistant isolates [25]. In the 1980s publications comparing $S$. epidermis and $S$. haemolyticus found increase in the number of methicillinresistant isolates of both species; although MR $S$. haemolyticus strains had a higher MIC [26]. A clear increase in MR-CoNS percentage was witnessed. The steep increase in CoNS infections was observed in a twenty year study in Switzerland as the rate of MR-CoNS isolates recovered from burn patients raised from $11 \%$ to 50 percent from 1986 to 2005 [27]. In the United States and four European countries, the proportion of CoNS resistant to oxacillin ranged from $51.4 \%$ in France to $75.2 \%$ in the United States while 2,905 CoNS isolates were examined in 2001 from SSTIs of hospitalized patients [28].

The mec genes are seen in the chromosome by a mobile SCCmec genetic feature. This cassette consists of three main elements: the mec gene complex, the ccr gene complex, and the joining area ('junkyard' or J). The mec gene complex comprises of the mecA gene itself, and the mecI (repressor), mecR1 (sensor inducer), and IS431mec insert sequence [29]. [30].

In our studies, we performed the antibiotic susceptibility test by disc diffusion method (Kirby-Bauer) for 20 isolates. All 20 isolates are resistant to methicillin $(5 \mu \mathrm{g})$ antibiotic. The 2 isolates are resistant to more than 2 antibiotics. Isolate number 6 resist methicillin, norfloxacin $(10 \mu \mathrm{g})$, and chloramphenicol $(30 \mu \mathrm{g})$. The isolate number 13 resists methicillin, norfloxacin $(10 \mu \mathrm{g})$, and ciprofloxacin $(5 \mu \mathrm{g})$. Multi-resistant CoNS isolates, displaying simultaneously resistance against at least three antibiotic classes, were prevalent.

The CoNS S. epidermidis strain was resistant to 6 distinct antibiotics (AMP, CRO, K, OB, OFX, and OT) [31].

In another study, 16 (29\%) staphylococci isolates were resistant to 3 or more antibiotics [32]. In 25 percent of the staphylococcal isolates from patients with chronic blepharitis, some others observed resistance to two or more antibiotics. Multiple antibiotic resistance can be seen as a reaction to extended therapy. Therefore, it is a cause for alarm to detect one strain immune to all antibiotics tested. These strains are a formidable challenge to hospitalized immune-compromised patients. Multidrug resistant CoNS often colonizes the skin of hospital personnel thus exposing vulnerable patients to life threatening infections [33].

Efflux pumping inhibitors (EPIs) may be a powerful strategy to overwhelm MDR. 1-(1-naphthyl-methyl)piperazine (NMP) and phenylalanine-arginine $\hat{\mathrm{I}} 2$ naphthylamide (PAÎ2N) are model EPIs that can block the activity AcrB, a large Escherichia coli efflux pump, or related homologous resistance pumps -nodulation-cell class division [34].

In Staphylococcus spp. efflux pumps is an important resistance mechanism. In addition to the chromosome pump and the plasmid-encoded NorA pump, which has been described previously, S. aureus chromosomic pumps include AbcA, NorB, NorC, MepA, MdeA, SdrM and Tet, SmR, and Sav-1866 type MFS [35, 36].

In the present study, the phylogenetic tree proves the SepA of $P$. putida is homologous with the various efflux proteins seen in Staphylococcus sp. And Vibrio sp.

\section{Conclusion}

Currently, antimicrobial resistance microorganisms have increased rampantly worldwide, which is a serious threat to humans and other animals. World Health Organization declared anti-microbial resistance as a catastrophe. These antimicrobial resistance pathogens are frequently found in different environments and often related to anthropogenic activities. Sewage treatment plants are treating and recycling the used contaminated waters. This recycled water is used for again human use. If those multiple drug resistant organisms are present after treatment, this will cause severe threat to human beings. So, we need to develop a modern treatment procedure for those sewage treatment plants that will effectively reduce multiple drug pathogens and organisms. The RND efflux proteins help to resist the antibiotics; they are superfamily efflux proteins involved in multiple drug-resistance. The widespread presence of methicillin resistance in CoNS is a cause for grave concern as sewage treatment plants are reservoirs for the spread of antibiotic resistance. Our study has shed light on the extensive presence of methicillin- CoNS in local sewage treatment plant.

\section{Conflict}

The authors declare that they have no competing interests.

\section{Funding}

Self-funded project.

\section{Acknowledgements}

Authors acknowledge the Post Graduate Department of Microbiology, The American College, Madurai for laboratory facilities and encouragement.

\section{References}

[1] Mangi, Shahida \& Hussain, Anwar \& Qazi, Muneer \& Kanhar, Nisar. (2016). Incidence of multidrug-resistant coagulasenegative Staphylococci in clinical samples of Tertiary care hospitals of Khairpur and Sukkur cities of Pakistan. International Journal of Biosciences. 9. 261-269. 10.12692/ijb/9.4.261-269.

[2] Abulreesh, H. H. (2011). Multidrug-resistant staphylococci in the environment. In International Conference on Biotechnology and Environment Management (Vol. 18, pp. 1$6)$.

[3] Jevons, M. P., \& Parker, M. T. (1964). The evolution of new hospital strains of Staphylococcus aureus. Journal of clinical $\begin{array}{llll}\text { pathology, } & 17 & 3), & 243-250 .\end{array}$ https://doi.org/10.1136/jcp.17.3.243. 
[4] Gopalakrishnan, R., \& Sureshkumar, D. (2010). Changing trends in antimicrobial susceptibility and hospital-acquired infections over an 8 years period in a tertiary care hospital in relation to introduction of an infection control programme. The Journal of the Association of Physicians of India, 58 Suppl, 25-31.

[5] Patel, A. K., Patel, K. K., Patel, K. R., Shah, S., \& Dileep, P. (2010). Time trends in the epidemiology of microbial infections at a tertiary care center in west India over last 5 years. J Assoc Physicians India, 58 (58), 37-40.

[6] D'Souza, N., Rodrigues, C., \& Mehta, A. (2010). Molecular characterization of methicillin-resistant Staphylococcus aureus with emergence of epidemic clones of sequence type (ST) 22 and ST 772 in Mumbai, India. Journal of clinical microbiology, 48 (5), $1806-1811$. https://doi.org/10.1128/JCM.01867-09.

[7] Rathanin Seng, U. L. (2017). High prevalence of methicillinresistant coagulase-negative staphylococci isolated from a university environment in Thailand. International Microbiology, 20 (2), 65-73. doi: 10.2436/20.1501.01.286.

[8] CDC, Antibiotic/Antimicrobial resistance report in US (2019), Retrieved from;

https://www.cdc.gov/drugresistance/index.html.

[9] Martinez, J. L. (2006). Role of non-clinical environments in the selection of virulence and antibiotic resistance determinants in pathogenic bacteria. J. Biol. Sci, $6(1), 1-8$.

[10] Kümmerer, K., Al-Ahmad, A., \& Mersch-Sundermann, V. (2000). Biodegradability of some antibiotics, elimination of the genotoxicity and affection of wastewater bacteria in a simple test. Chemosphere, 40 (7), 701-710.

[11] Watkinson, Andrew \& Murby, E. J. \& Kolpin, Dana \& Costanzo, Simon. (2009). The occurrence of antibiotics in an urban watershed: From wastewater to drinking water. The Science of the total environment. 407. 2711-23. 10.1016/j.scitotenv.2008.11.059.

[12] Volkmann, H., Schwartz, T., Bischoff, P., Kirchen, S. and Obst, U. (2004) Detection of clinically relevant antibiotic resistance genes in municipal wastewater using real-ime PCR (Taqman). J Microbiol Methods 56, 277-286.

[13] Börjesson, S., Matussek, A., Melin, S., Löfgren, S., \& Lindgren, P. E. (2010). Methicillin-resistant Staphylococcus aureus (MRSA) in municipal wastewater: an uncharted threat?. Journal of applied microbiology, 108 (4), 1244-1251. https://doi.org/10.1111/j.1365-2672.2009.04515.x.

[14] Cyprowski M, Szarapińska-Kwaszewska J, Dudkiewicz B, Krajewski JA, Szadkowska-Stańczyk I (2005) Exposure assessment to harmful agents in workplaces in sewage plant workers. Med Pr 56 (3): 213-222.

[15] Gerardi MH, Zimmerman MC (2005) Wastewater pathogens. Wiley, New Jersey.

[16] Becker, K., Heilmann, C., \& Peters, G. (2014). Coagulasenegative staphylococci. Clinical microbiology reviews, 27 (4), 870-926.

[17] Longauerova, A. (2006). Coagulase negative Staphylococci and their participation in pathogenesis of human infections. Bratislavské lekárske listy. 107. 448-52.

[18] Kondapaneni, Saileela \& Vanaparthi, Nagaraju \& Anukolu,
Ravishankarreddy \& Kumar, A. N. (2017). Biochemical Characterization and Antibiotic Susceptibility of Coagulase Negative Staphylococci Isolates from Exudates and Body Fluids. Indian Journal of Mednodent and Allied Sciences. 5. 10.5958/2347-6206.2017.00012.7.

[19] Dinges, M. M.; Orwin, P. M.; Schlievert, P. M. Exotoxins of Staphylococcus aureus. Clin. Microbiol. Rev. 2000, 13, 16-34.

[20] Cotar, Ani \& Chifiriuc, Mariana \& Dinu, Sorin \& Bucur, Marcela \& Iordache (Curutiu), Carmen \& Banu, Otilia \& Dracea, Olguta \& Larion, Cristina \& Lazar, Veronica. (2010). Screening of Molecular Virulence Markers in Staphylococcus aureus and Pseudomonas aeruginosa Strains Isolated from Clinical Infections. International journal of molecular sciences. 11. 5273-91. 10.3390/ijms11125273.

[21] Lakshmi, H. P., Prasad, U. V., Yeswanth, S., Swarupa, V., Prasad, O. H., Narasu, M. L., \& Sarma, P. V. (2013). Molecular characterization of $\alpha$-amylase from Staphylococcus aureus. Bioinformation, 9 (6), 281-285. https://doi.org/10.6026/97320630009281.

[22] Moraveji, Z., Tabatabaei, M., Shirzad Aski, H., \& Khoshbakht, R. (2014). Characterization of hemolysins of Staphylococcus strains isolated from human and bovine, southern Iran. Iranian journal of veterinary research, 15 (4), 326-330.

[23] Kresken M, Hafner D. 1999. Drug resistance among clinical isolates of frequently encountered bacterial species in central Europe during 1975-1995. Infection 27 (Suppl 2): S2-S8. doi: 10.1007/BF02561661.

[24] Hamilton-Miller JM, Iliffe A. 1985. Antimicrobial resistance in coagulase-negative staphylococci. J. Med. Microbiol. 19: 217-226. doi: 10.1099/00222615-19-2-217.

[25] Guggenheim M, Zbinden R, Handschin AE, Gohritz A, Altintas MA, Giovanoli P. 2009. Changes in bacterial isolates from burn wounds and their antibiograms: a 20-year study (1986-2005). Burns 35: 553-560. doi: 10.1016/j.burns.2008.09.004.

[26] Jones ME, Karlowsky JA, Draghi DC, Thornsberry C, Sahm DF, Nathwani D. 2003. Epidemiology and antibiotic susceptibility of bacteria causing skin and soft tissue infections in the U.S.A. and Europe: a guide to appropriate antimicrobial therapy. Int. J. Antimicrob. Agents 22: 406-419. doi: 10.1016/S0924-8579(03)00154-7.

[27] Tesch W, Ryffel C, Strässle A, Kayser FH, Berger-Bächi B. 1990. Evidence of a novel staphylococcal mec-encoded element (mecR) controlling expression of penicillin-binding protein 2'. Antimicrob. Agents Chemother. 34: 1703-1706. doi: 10.1128/AAC.34.9.1703.

[28] Hiramatsu K, Katayama Y, Yuzawa H, Ito T. 2002. Molecular genetics of methicillin-resistant Staphylococcus aureus. Int. J. Med. Microbiol. 292: 67-74. doi: 10.1078/1438-4221-00192.

[29] Virdis, S., Scarano, C., Cossu, F., Spanu, V., Spanu, C., \& De Santis, E. P. (2010). Antibiotic Resistance in Staphylococcus aureus and Coagulase Negative Staphylococci Isolated from Goats with Subclinical Mastitis. Veterinary medicine international, 2010, 517060. https://doi.org/10.4061/2010/517060.

[30] Pinna A, Zanetti S, Sotgiu M, et al., Identification and antibiotic susceptibility of coagulase negative staphylococci isolated in corneal/external infections. British Journal of Ophthalmology 1999; 83: 771-773. 
[31] Archer GL, Climo MW. Antimicrobial susceptibility of coagulase-negative staphylococci. Antimicrob Agents Chemother 1994; 38: 2231-7.

[32] Soparkar, K., Kinana, A. D., Weeks, J. W., Morrison, K. D., Nikaido, H., \& Misra, R. (2015). Reversal of the Drug Binding Pocket Defects of the AcrB Multidrug Efflux Pump Protein of Escherichia coli. Journal of bacteriology, 197 (20), 3255-3264. https://doi.org/10.1128/JB.00547-15.

[33] Otto, M. (2013). Coagulase-negative staphylococci as reservoirs of genes facilitating MRSA infection. Bioessays 35, $4-11$.
[34] Xu Z, M. H. (2015). Antibiotic resistance and mecA characterization of coagulase-negative staphylococci isolated from three hotels in London, UK. Front Microbiol, 6, 947. doi: 10.3389/fmicb.2015.00947.

[35] Zhang M, O. M. (2011). Prevalence of antiseptic-resistance genes in Staphylococcus aureus and coagulase-negative staphylococci colonising nurses and the general population in Hong Kong. J Hosp Infect., 78 (2), 113-117. doi: 10.1016/j.jhin.2011.02.018.

[36] Li, X. Z., \& Nikaido, H. (2009). Efflux-mediated drug resistance in bacteria: an update. Drugs, 69 (12), 1555-1623. https://doi.org/10.2165/11317030-000000000-00000. 